\title{
Deep searches for isolated radio-quiet neutron stars
}

\author{
Joachim Trümper \\ Max-Planck-Institut für Extraterrestrische Physik, 85740 Garching, Germany
}

\begin{abstract}
Using ROSAT seven isolated neutron stars have been discovered characterised by blackbody spectra at temperatures of 400-800 thousand $\mathrm{K}$ and the absence of radio and high energy emissions. Observations with Keck, HST and VLT show faint optical counterparts ( $\mathrm{m} \sim$ 24-29) with Rayleigh-Jeans-type spectra $\left(\sim \nu^{2}\right)$ and large proper motions. These objects are believed to be cooling neutron stars. They are of great interest from the point of view of neutron star astrophysics and nuclear physics. In particular, they allow us to measure neutron star radii and thus to constrain the equation of state at very high densities. We discuss the possibility of discovering larger samples of these objects with future sensitive X-ray sky surveys and follow-up optical observations with extremely large telescopes.
\end{abstract}

\section{Introduction}

The bright non-thermal X-ray emission of pulsars as well as the strong thermal emission from the accretion disks or columns of neutron stars in binary X-ray sources were discovered already in the sixties. Studies using rocket or balloon experiments and various satellites from UHURU to RXTE have revealed a wealth of information about neutron star properties like masses, magnetic moments and surface magnetic fields. The discovery of thermal emission from the hot photospheres of (cooling) neutron stars became possible in an indisputable way first with ROSAT because of it's high sensitivity in the soft X-ray band (0.1-1 keV). Later observations with Chandra and XMM-Newton have added many important facets to the subject due to the enhanced sensitivity and spectral resolution of their instruments. So far, the photospheric emission of neutron stars has been detected from

- pulsars in addition to the broadband non-thermal emission from the magnetosphere

- polar caps in pulsars or millisecond pulsars heated by cooling from inside or heating by high energy particle bombardment

- neutron stars which do not show radio or high energy emission and which are not associated with supernova remnants.

This latter class of objects - radio-quiet isolated neutron stars - is the subject of this paper. They represent neutron stars in their cleanest form; we see them because they are hot - just like ordinary stars.

\section{Radio-quiet isolated neutron stars}

At present we know seven of these sources which all have been discovered with ROSAT. The first and most prominent one, RX J1856-3754, was detected in a serendipitous observation (Walter et al. 1996), the others were found in the all sky survey. The basic properties of these "The Magnificent Seven" (Treves et al. 2001) are listed in Table 1 and can be summarized as follows:

- blackbody-like soft X-ray spectra, temperatures of 0.5-1.3 MK

- no radio or high energy emission of magnetospheric origin 
- low interstellar absorption $\left(\mathrm{n}_{H} \sim 10^{20} \mathrm{~cm}^{-2}\right)$, large proper motions

- long periodicities compared with radio pulsars

- no obvious association with supernova remnants

- faint optical counterparts, large ratios of X-ray/optical flux

Because of their low $\mathrm{n}_{H}$ and their large proper motions these objects must have distances of at most a few hundred pc. The parallax obtained with HST for RX J1856-3754 yields $117 \pm 12$ pc (Walter \& Lattimer 2002) or a somewhat larger distance (Kaplan 2004). The pulsations show a low amplitude (pulsed fraction of $\sim 10 \%$ ), as expected from a star with an inhomogeneous temperature distribution. RX J0720-3125 is spinning down, which leads to an estimate of its magnetic field of a few $10^{13}$ Gauss. Proton cyclotron absorption lines observed in four of these objects yield magnetic fields of the same order (Haberl 2005).

Table 1. X-ray and optical properties of the seven radio-quiet isolated neutron stars (Haberl 2005).

\begin{tabular}{lcrlc}
\hline Object & $k T / \mathrm{eV}$ & $P / \mathrm{s}$ & Optical & proper motion \\
\hline RX J0420.0-5022 & 44 & 3.45 & $\mathrm{~B}>26.6$ & - \\
RX J0720.4-3125 & $85-95$ & 8.39 & $\mathrm{~B}=26.6$ & $97 \mathrm{mas} / \mathrm{y}$ \\
RX J0806.4-4123 & 96 & 11.37 & $\mathrm{~B}=24$ & - \\
RX J1308.5+2127 & 86 & 10.31 & $m_{50 c c d}=26.6$ & - \\
RX J1605.3+3249 & 96 & - & $\mathrm{B}=27.2$ & $145 \mathrm{mas} / \mathrm{y}$ \\
RX J1856.5-3754 & 60 & - & $\mathrm{V}=25.7$ & $332 \mathrm{mas} / \mathrm{y}$ \\
RX J2143.0+0654 & 100 & 9.43 & $\mathrm{R}>23$ & - \\
\hline
\end{tabular}

\section{Astrophysical and physical significance of the radio-quiet isolated neutron stars}

Obviously these stars represent a local population in our galaxy. Because of their large proper motions they cannot be heated by accretion from the interstellar medium. Therefore they must be cooling. Their temperatures indicate cooling ages of a few hundred thousand years which explains why they are not associated with supernova remnants. They have either been born with long periods or spun down to their present periods which requires magnetic fields of $>10^{13}$ Gauss - in agreement with the observed values. As (almost) isotropic emitters they are ideally suited for determining the local space density of neutron stars.

These objects can also be used to constrain the equation of state (EOS) of matter at high densities by measuring their radiation radii. This requires an accurate knowledge of the X-ray and optical spectrum and of the stellar distance. So far this method could be applied only to RX J1856-3754 due to its known parallax. The high resolution Chandra LETG data of this source (Burwitz et al. 2003), combined with the optical data can be fit either by a two temperature model or a model with a continuous temperature distribution over the stellar surface (Fig. 1 ). In both cases the resulting radiation radius $R_{\infty} \sim 16.8$ $\mathrm{km}$, which can be regarded as a lower limit because it is based on blackbody fits (Trümper et al. 2004; Trümper 2005). Such a large radius requires a very stiff equation of state. The same conclusions were reached by Braje \& Lattimer (2002) and Walter \& Lattimer (2002) on the basis of low resolution ROSAT PSPC spectra. 

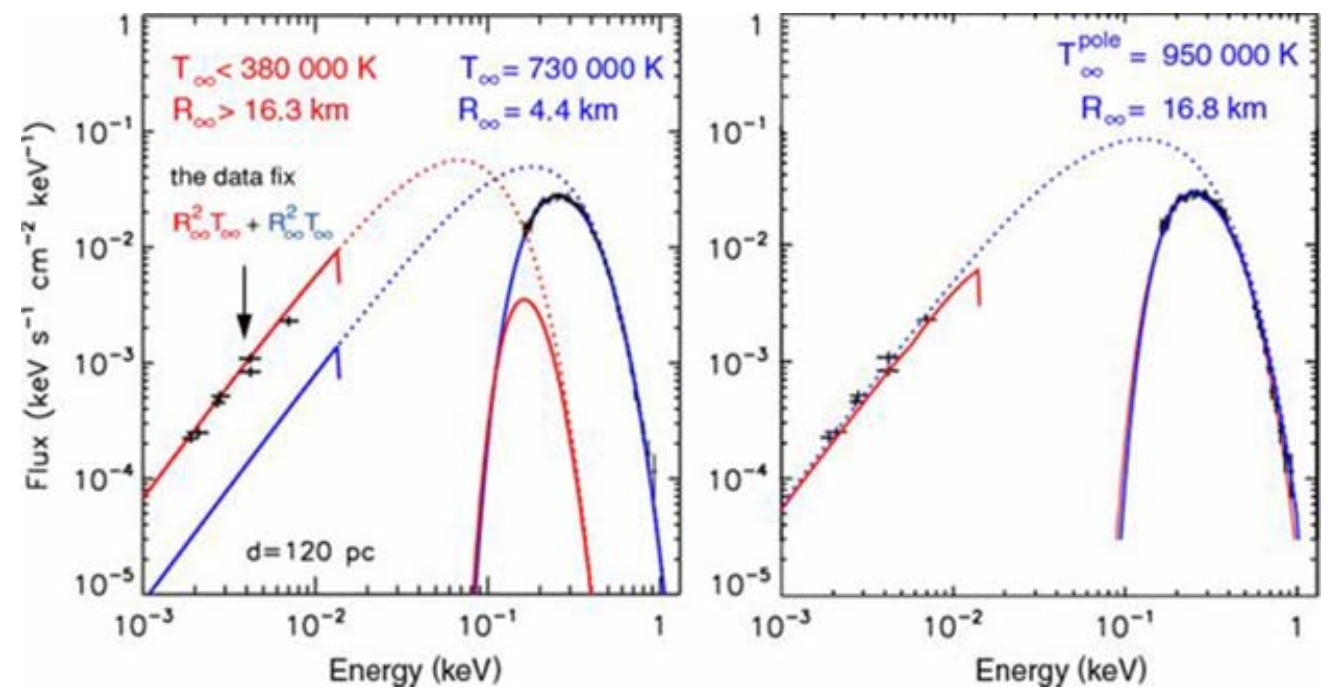

Figure 1. The X-ray and optical spectrum of RX J1856-3754 is well fit by a two-component blackbody spectrum (left) or a blackbody spectrum from star showing a continuous temperature distribution between pole and equator (Trümper et al. 2004; Trümper 2005).

Left: a two-component blackbody fit with a radius $\mathrm{R}_{\infty} \sim 16.9 \mathrm{~km} \times d_{120}$.

Right: a blackbody fit with a continuous temperature distribution $T=T_{\text {pole }}\left[1+\left(\theta / \theta_{0}\right)^{2}\right]^{-1}$ requires a radius of $16.8 \mathrm{~km} \times d_{120}$.

\section{Search criteria and strategies}

To increase the number of known radio-quiet isolated neutron stars one needs first of all a more sensitive all sky survey in soft X-rays. The planned ROSITA would have a factor of $\sim 10$ larger sensitivity, and an improved energy resolution (factor $\sim 4$ ) compared with ROSAT (Hasinger 2005). Assuming a homogeneous spatial distribution of sources would lead to an increase in the number by a factor of $10^{3 / 2} \sim 30$, viz. about 200 objects. In reality this number will be somewhat lower because of the interstellar absorption and the finite galactic scale height of neutron stars. Since the X-ray survey only yields candidates, the neutron stars among them have to be identified by optical follow-up observations, which can be done in three steps - using existing optical sky surveys, observations with telescopes of the 3-5 m class, and finally observations with ELTs. Primarily photometric observations in different colours would suffice since the optical spectra are of the RayleighJeans type. However, spectroscopic measurements might reveal interesting information about the photosphere.

RX J0420-5022 has probably a magnitude $\mathrm{m}>29$. A sensitivity increase of a factor 10 then requires that the optical work on ROSITA sources has to extend to $\mathrm{m} \sim 32$. Extrapolating the data of the Hubble Deep Field North one finds that the number of galaxies at $\mathrm{m} \leqslant 32$ is about one in an error circle of 1 arcsec radius. Since the ROSITA sky survey will have a positional accuracy of only 10-20 arcsec pointed observations with Xray telescopes like Chandra or XEUS ( $\sim 1$ arcsec positions) are required to obtain reliable identifications. In addition, radio-quiet isolated neutron stars can be distinguished from background galaxies because they are point sources and show a $\sim \nu^{2}$-spectrum in the optical range. 


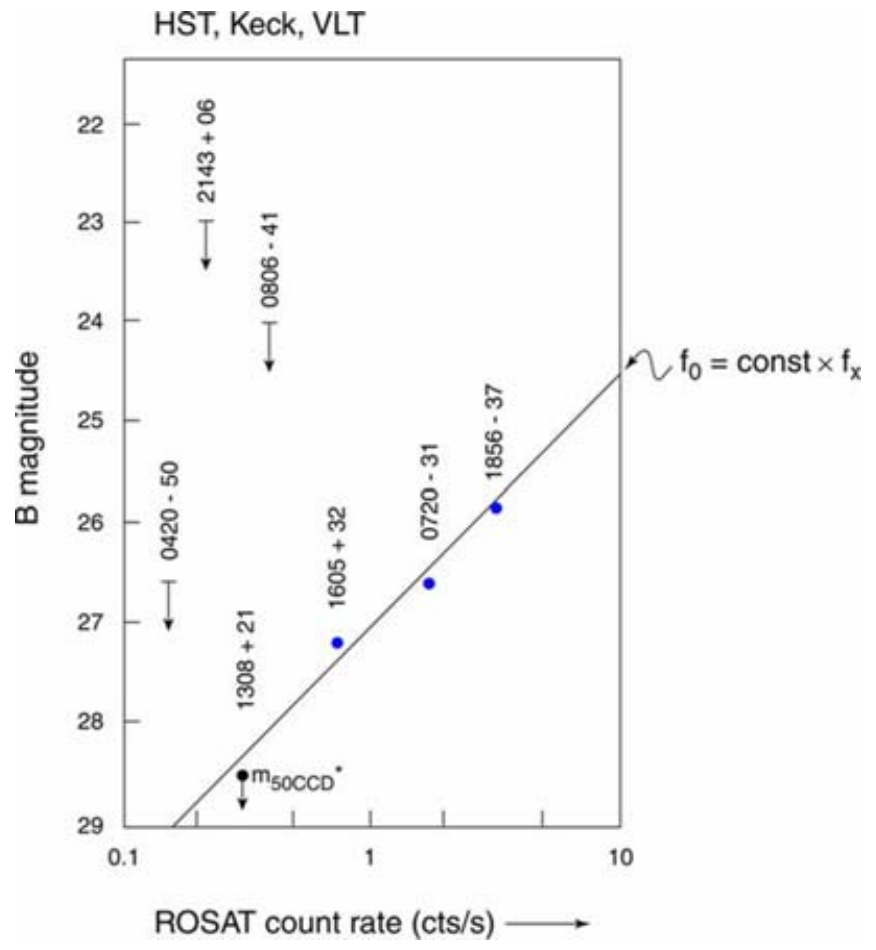

Figure 2. The $f_{o} / f_{x}$ plot of the Magnificent Seven suggests that so far only the three brightest $\mathrm{X}$-ray sources have been optically identified for sure (these are also the only ones showing large proper motions).

\section{Conclusions}

Using ROSITA and Chandra/XEUS and optical telescopes of the ELT class it should be possible to increase the number of radio-quiet isolated neutron stars up to a few dozen. This would be very important for neutron star astrophysics and nuclear physics.

\section{Acknowledgements}

I gratefully acknowledge useful discussions with Vadim Burwitz and Frank Haberl.

\section{References}

Braje, T.M. \& Lattimer, R.W. 2002, ApJ 580, 1043-1047

Burwitz, V., Haberl, F., Neuhaeuser, R., Predehl, P., Trümper, J. \& Zavlin, V.E. 2003, A\&A $397,557-564$

Haberl, F. 2005, in: U.G. Briel, S. Sembay \& A. Read (eds.), Proceedings of the 2005 EPIC Consortium Meeting, MPE Report 288

Hasinger, G. 2005, private communication

Kaplan, D.K. 2004, private communication

Treves, A., Popov, S.B., Colpi, M., Prokherov, M.E. \& Turolla, R. 2001, in: R. Giacconi, L. Stella \& S. Serio (eds.) X-Ray Astronomy 2000, ASP Conf. Series, Vol. 234, 225

Trümper, J. 2005, in: A. Baykal et al. (eds.), The Electromagnetic Spectrum of Neutron Stars (NATO Science Series, II), Springer, Vol. 210, p. 117

Trümper, J., Burwitz, V., Haberl, F. \& Zavlin, V.E. 2004 The Restless High-Energy Universe, Nuclear Physics B Proceedings Supplements, Vol. 132, p. 560

Walter, F.M., Wolk, S.J. \& Neuhaeuser, R. 1996, Nature 379, 239

Walter, F.M. \& Lattimer, J. 2002, ApJ 576, L145-148 


\section{Discussion}

MoulD: Are there model atmospheres for the photospheres of these stars?

TRÜMPER: Yes. There are atmospheric models for light (H, He) up to heavy elements $(\mathrm{Fe})$ for the non-magnetic case $\left.\left(B<10^{9} \mathrm{G}\right)\right)$. For high magnetic fields only $\mathrm{H} / \mathrm{He}$ atmospheres have been calculated. For Fe and other heavy elements the atomic physics (cross sections, energy levels, etc.) are available only in crude approximations. The X-ray spectrum of RXJ 1856, as measured with the Chandra LETG $(\lambda / \Delta \lambda)$, does not show any lines/absorption edges. It is a perfect blackbody. It is highly inconsistent with a non-magnetic Fe atmosphere or solar composition atmosphere. A non-magnetic $\mathrm{H}$ or $\mathrm{He}$ atmosphere over-predicts the optical spectrum by a large amount.

There are two explanations for the featureless blackbody spectrum of RXJ 1856: (1) A heavy element atmosphere with lines/edges washed away by strong magnetic shifts. (There is evidence that these radio-quiet isolated neutron stars have magnetic fields of $10^{13} \mathrm{G}$ Which would lead to huge shifts). (2) Alternatively the star may have no atmosphere, but a condensed matter surface. 